\title{
Forecasting Full-Path Network Congestion Using One Bit Signalling
}

\author{
Mussie Woldeselassie \\ Department of E\&EE \\ University College London \\ Torrington Place \\ London WC1E 7JE, UK \\ Email: mwoldese@ee.ucl.ac.uk
}

\author{
Richard G. Clegg \\ Department of E\&EE \\ University College London \\ Torrington Place \\ London WC1E 7JE, UK \\ Email: richard@richardclegg.org
}

\author{
Miguel Rio \\ Department of E\&EE \\ University College London \\ Torrington Place \\ London WC1E 7JE, UK \\ Email: m.rio@ee.ucl.ac.uk
}

\begin{abstract}
In this paper, we propose a mechanism for packet marking called Probabilistic Congestion Notification (PCN). This scheme makes use of the 1-bit Explicit Congestion Notification (ECN) field in the Internet Protocol (IP) header. It allows the source to estimate the exact level of congestion at each intermediate queue. By knowing this, the source could take avoiding action either by adapting its sending rate or by using alternate routes. The estimation mechanism makes use of time series analysis both to improve the quality of the congestion estimation and to predict, ahead of time, the congestion level which subsequent packets will encounter.

The proposed protocol is tested in ns-2 simulator using a background of real Internet traffic traces. Results show that the methods can successfully calculate the congestion at any queue along the path with low error levels.
\end{abstract}

Index Terms-TCP, Congestion Control, ECN, Forecasting.

\section{INTRODUCTION}

Today's packet based Internet Protocol (IP) relies on the congestion control in Transmission Control Protocol (TCP) for stability [1]. TCP congestion mechanisms react to congestion by adjusting a "congestion window" according to whether packets are received or lost. Many variants of TCP have been proposed which alter either the means by which congestion is detected or the response to that congestion. The key contribution of this paper is twofold. Firstly, a new protocol Probabilistic Congestion Notification (PCN) is proposed for probabilistic packet marking which uses only a single bit, the Explicit Congestion Notification (ECN) bit in the IP header [2]. In PCN, routers do not maintain any per-flow state of the flows. The scheme allows an end host to estimate not only the level of congestion that packets encountered but also to track the levels of congestion separately for each of the intervening routers. Congestion information from all routers will be useful in several scenarios like multi-path TCP, overlay peer selection or future user selectable routing schemes, for further motivation see [3]. At its simplest, taking the largest such estimate along the path can be used to estimate the bottleneck link.

Secondly, time series analysis methods (see Box-Jenkins [4] further details) are used to both improve the accuracy of this estimate and to allow the level of congestion to be predicted. This allows end hosts to decide on their actions based upon the level of congestion a packet will encounter if it is sent now rather than the level it would have encountered had it been sent at some previous time.

In order to test the protocol and prediction real traffic traces have been used to provide realistic background traffic. The results show that PCN can produce a reliable estimate for the true congestion level on all routers with low root mean square error and bias. Without the time series analysis correction, PCN has much higher errors unless long sampling times are used. The scheme described predicts the level of congestion at all routers between two end hosts.

\section{A. Background}

Router based packet marking schemes are based upon one common idea, that routers notify end hosts of congestion by modifying one (or more) bits in the packet header [5]. Examples include the Random Exponential Marking (REM) [6], Random Additive Marking (RAM) [7], Deterministic Quantisation Marking (DQM) [8], Variable-structure Congestioncontrol Protocol (VCP) [9] and eXplicit Congestion Protocol (XCP) [3]. The schemes can be differentiated from each other by whether the marking is deterministic or probabilistic, by how many bits in the header they use and by whether they attempt to calculate congestion on the whole path or on individual routers along a path (see section $\Pi-\mathrm{I}$ for further detail)

Different schemes vary how the level of congestion is estimated. In one common scheme (used by VCP amongst others) the router estimates its level of congestion using the load factor. The load factor is described in [10], it is an estimate of current local congestion which is tracked by every router that is capable of the packet marking scheme. The load factor is estimated for each outgoing link for intervals of size $t_{\rho}$. The value $t_{\rho}$ should be larger than the round-trip time (RTT) of most flows, but small enough to capture the dynamic changes in traffic level [9]. In this paper $t_{\rho}$ of $200 \mathrm{~ms}$ is used since this is the value suggested in [9]. The load factor within period $l$ is given by

$$
\rho_{l}=\frac{\lambda_{l}+\kappa_{q} \hat{q}_{l}}{\gamma_{l} C_{l} t_{\rho}}
$$


where, $\lambda_{l}$ is the amount of input traffic (number of packets) during period $l, \hat{q}_{l}$ is the persistent queue length during period $l$ (measured with a low pass filter), $\kappa_{q}$ controls how fast the persistent queue drains, $\gamma_{l}$ is the target utilisation (set to a value close to 1) and $C_{l}$ is outgoing link capacity. For further details of the precise formulation of these quantities see [10].

\section{THE PROPOSED PACKET MARKING SCHEME}

This section describes the new packet marking scheme, probabilistic congestion notification (PCN). This scheme uses only a single bit, does not require per-flow state at the router and produces an estimate for the congestion level at each queue the traffic encounters on its outward journey.

\section{A. Protocol at router and end hosts}

The PCN scheme marks a single bit in the packet header, this is used with statistical methods to estimate the congestion of any of several possible intermediate routers. PCN relies on two fields in the IP header: the IP identifier (IPid) and the time to live (TTL) fields. These are initialised by the original source of the packet and the TTL field is reduced by one at every hop on the path.

The marking scheme at the router is both stateless and extremely simple. Assume each intermediate router has a current load factor (1) which the source wishes to estimate. The load factor $\left(\rho_{l}\right)$ is in the range $[0,100]$ (values above 100 are rounded down). This range is not a necessary assumption of the algorithm as discussed later.

Assume that there are at most $M$ intervening routers initially this is fixed at 32 for all connections. It is not a problem for the algorithm if there are fewer intervening routers (but it does cause loss of efficiency). If there are more then some information will be split between routers (see later discussion). The strategy is to allow each outgoing packet to have its ECN bit set by at most one of the intervening routers. This ECN bit is set in a probabilistic manner governed by the load factor in such a way that the ratio of marked packets to total packets is equal to the load factor (divided by 100). Whether the ECN bit is marked or not marked is communicated back to the source on the acknowledgement (ACK) for the outgoing packet (so the receiver simply has to copy the state of the ECN bit onto the ACK). The IPid and TTL are used to determine which router can mark the packet.

Consider the condition

$$
\text { TTL } \bmod M=\text { IPid } \bmod M .
$$

By definition the IPid remains constant and TTL decreases by one at each hop. Therefore, if there are at most $M$ intervening routers then for only one such router will this condition be true. Only the router for which (2) is true may set the ECN bit on a packet. Define a packet as markable by router $i$ if (2) is true for that packet for router $i$. The source knows the IPid and TTL for every packet and can calculate for which router a given packet was markable.

The router for which condition (2) is met marks the packet with a probability equal to the load factor. The expectation value of the proportion of markable packets with the ECN bit set by router $i$ is equal to its load factor (while the load factor remains constant - see section $\Pi \mathrm{II}-\mathrm{C}$ for more discussion of this issue). Therefore, the proportion of ACKs from packets markable by router $i$ which have their ECN bit set is an unbiased estimate of the load factor.

The full protocol for PCN can be simply given as follows.

- The source sets the ECN bit to zero, TTL sets to $M$, and increments the IPid by one for each packet.

- Intermediate routers, if condition (2) is met, set the ECN bit with a probability equal to their load.

- The receiver copies the ECN bit from a packet onto that packet's ACK (there must be one ACK for every packet).

- The source tracks the ECN bits on ACKs to estimate congestion on intermediate routers.

This algorithm replaced ECN at routers and cannot coexist with it (routers performing standard ECN marking will confuse PCN estimates). It is this final part which enables the source to estimate congestion and which will be a main focus of the results in this paper. Section $\amalg-\mathrm{C}$ shows how time series modelling techniques can be used to improve the estimate of congestion level and section IV shows ns-2 modelling results which prove the scheme practical for realistic estimation scenarios.

The value $M=32$ was chosen since [11] shows that a hop count of more than 30 is extremely rare in the real Internet. However, if there are more than $M$ routers the protocol's failure mode is not a major issue although some packets may be marked by more than one router.

If there are less than $M$ intervening routers some packets are not markable by any and an opportunity to get data is lost. A possible improvement is to pre-signal the actual number of intervening routers (by communicating the TTL of the SYN packet). The PCN source can ensure that only packets which have condition (2) met for some intervening router are sent. This will increase the number of samples at each router. This improvement is also tested in section IV

Estimates of congestion other than that given by (1) can easily by used by PCN. If the new load equation is not in the range $[0,100]$ then a simple linear transform, $a l+b$, will map it into this range. If some regions of the range are more of interest than others then nonlinear transforms could be used.

\section{B. Comparison with other packet marking schemes}

It is important to recognise how PCN differs from other packet marking schemes recently suggested in the literature. Like XCP, PCN estimates the congestion level at each intervening queue. However, XCP requires a new 128 bit header to record the information whereas $\mathrm{PCN}$ requires only a single ECN bit. DQM (and its variants) use a similar scheme involving both TTL and IPid. However, they require a lookup table to be stored in each router in advance, it is a deterministic rather than a probabilistic scheme and requires two bits in the IP header. RAM like PCN is a probabilistic packet marking scheme. It also uses a single ECN bit to mark packets, but it attempts to calculate the load on the whole path rather than 
on each queue on the path separately. It uses the IP TTL field to estimate the number of routers in the network.

\section{Congestion estimation algorithm}

Let $\rho_{l}$ be the load factor in time period $l$ with $l \in\{0,1, \ldots\}$. Each time period is of length $t_{\rho}$ so $\rho_{l}$ is the estimate for time $\left[l t_{\rho},(l+1) t_{\rho}\right)$. The source estimates congestion every period $t_{P}$. Consider the source attempting to estimate the load at the $i$ th intermediate router. Let $e_{l}$ be the ratio of ECN marked ACKs to total ACKs (markable by a given intermediate router $i$ ) in the $l$ th such period $\left[l t_{P},(l+1) t_{P}\right)$. If this time period is short then insufficient packets will be received to get a good estimate. If it is too long, the estimate will not capture the dynamic nature of the traffic.

Let $L_{l}$ be the mean value of the load factor $\rho$ in the time period $\left[l t_{P},(l+1) t_{P}\right)$. Note that the load factor may have changed over this period, particularly if $t_{P}$ (the time scale over which load factor is estimated by the source) is significantly larger than $t_{\rho}$ the time scale over which the load factor is held constant. This problem is to some extent unavoidable without time synchronisation between source and router. For the real traffic traces investigated in this work the congestion level remained similar for much longer time scales than $t_{\rho}$ and $t_{P}$.

One possible estimate for $L_{l+1}$ is $e_{l}$. However, it is clear that if $L_{l}$ does not change too much between given time periods then $e_{l-1}, e_{l-2}, \ldots$ can provide additional information to help predict $L_{l+1}$. The approach taken here is to use the well-known Autoregressive Integrated Moving Average (ARIMA) models to provide an improved predictor [4]. If ARIMA models can be fitted to the time series $e_{l}$ this can be used to get a good prediction of $L_{l+1}$ (since $e_{l}$ is an unbiased estimate of $L_{l}$ as previously proved). The selection of an appropriate ARIMA model is discussed in section IV-A.

\section{Simulation Methodology}

The protocol has been implemented using the simulation tool ns-21. Real life traffic traces collected by CAIDA have been used to create realistic background traffic (critically important for testing the ARIMA model). The simulation topology was chosen with the following points in mind: 1) The congestion levels should arise from realistic traffic 2) The (artificially generated) PCN flows should not significantly contribute to this congestion level 3) The congestion at a given router should be a product of the mixing of more than one traffic source.

The parking-lot topology used for the simulations is shown in figure 1 The two hosts labelled Ns_agent, each sends PCN enabled traffic to each other. For the purpose of congestion response in the simulation, the traffic behaves exactly like TCP New-Reno but the congestion marking from PCN is recorded. This PCN traffic crosses five intervening routers before reaching its destination. Six flows derived from real traffic traces are used to provide background traffic. These

\footnotetext{
${ }^{1}$ Network Simulator (ns-2), http://www.isi.edu/nsnam/ns
}

flows are between the hosts prefixed with Real on the diagram.

Two separate experiments are done using data derived from the CAIDA project OC-12 2 and OC- $48 \sqrt[3]{3}$ traffic traces. The traces known as OC-12-1500 and OC-48-0900-0 are used, both hour long traces. In order to get six real traces which have synchronised time behaviour, the CAIDA traces were each split by source IP address into six separate traces. For the first experiment the six data files from the OC48 contained 79,550,409 packets and those from the OC-12 contained 17,840,896 packets. This approach is taken because these traces will then be correlated in time (traces from $9 \mathrm{am}$ and $2 \mathrm{am}$ local time might exhibit very different traffic behaviour). Other traffic traces from these data sets have been tested with similar results to those reported in the next section.

UDP packets with length and inter-packet delay specified in these six traces are then fed into the network at each of the hosts suffixed src_n. Although obviously an "open-loop" simulation like this does not capture the responsive nature of TCP, the authors consider it a more realistic situation than using artificially generated TCP where the arrival behaviour might be extremely unrealistic and hence unrealistically easy to predict. This issue is, of course, extremely important when considering the utility of an ARIMA model.

Each router calculates its load factor at $200 \mathrm{~ms}$ intervals, as described in (1). The PCN hosts collect statistics at intervals of length $t_{P}$ (this interval varies and $t_{P}>200 \mathrm{~ms}$ ) and calculate the proportion of marked and unmarked packets for each intervening router which could have marked them. This proportion provides an estimate of the mean load factor over the period $t_{P}$. A better estimate for the mean load factor in the next time period can then be obtained using the time series forecasting as described in section II-C $t_{P}$ varies and the actual load factors at each router and the predicted load factor at the hosts is recorded for each of such intervals.

\section{Simulation Results}

In all the experiments in this section, the first $10 \%$ of the traffic is discarded as ns-2 warms up and the second $10 \%$ is used to estimate parameters for the time series model. As previously discussed, this load factor changes every $t_{\rho}$ seconds (0.2 seconds). Each PCN source produces an estimate for congestion at each outgoing queue every $t_{P}$ seconds. The time period $t_{P}$ varies between 0.2 and 3.2 seconds. This allows investigation of PCN behaviour as the number of samples per time period changes. The predictor $\hat{L_{i+1}}$ is compared with the crude estimate obtained by assuming the load factor to be $e_{i}$, the proportion of marked ACKs in the previous time period.

\section{A. Determining the appropriate prediction model}

A general $\operatorname{ARIMA}(p, d, q)$ model could be thought of as combining an autoregressive $\operatorname{AR}(p)$ component, a moving average $\mathrm{MA}(q)$ component and a differencing component $d$ - for details see [4] amongst many others. The first task is to

\footnotetext{
${ }^{2} \mathrm{OC12}$, www.caida.org/data/passive/passive_2007_dataset.xml

${ }^{3}$ OC48, www.caida.org/data/passive/passive_oc48_dataset.xml
} 


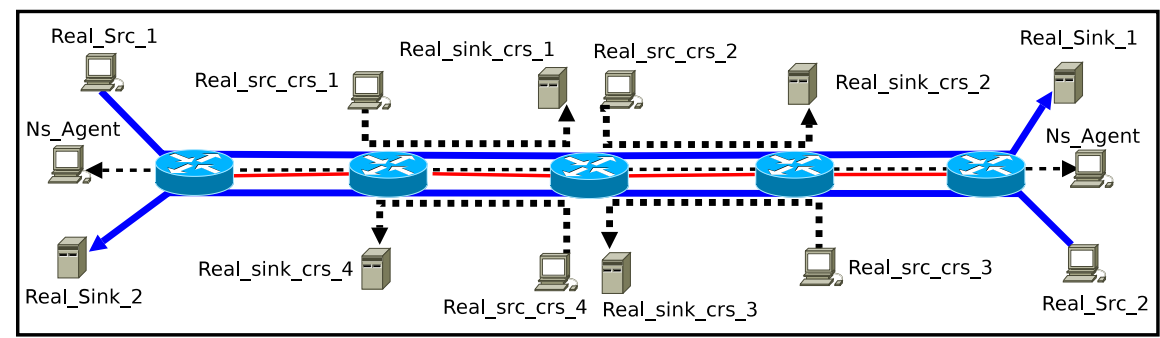

Fig. 1. Topology used for ns-2 simulation

determine the order of the model, that is the values of $p, d$ and $q$. For a given time series, in order to determine the order of the appropriate ARIMA, the autocorrelation function (ACF) and partial autocorrelation function (PACF), both functions of lag $k$, are examined. If the $\mathrm{ACF} \rho(k)$ becomes insignificant for $k>q$ for some small $q$ then an $\operatorname{AR}(q)$ model may be the best fit. Similarly, if the PACF $P(k)$ becomes insignificant for $k>p$ and some small $p$ then an $\operatorname{AR}(p)$ model may be effective. In the case of the $e_{i}$ time series examined here, in all cases considered, the ACF of the differenced series $d_{i}=e_{i+1}-e_{i}$ fell off quickly. This can be seen in figure 2 (the dotted lines indicate a 95\% confidence interval - results between them are likely to be merely noise) indicating only the lag one component is very significant (the lag zero component is by definition one). This is an extremely strong indicator than an $\operatorname{ARIMA}(0,1,1)$ model is appropriate. Other ARIMA models were tested but the $\operatorname{ARIMA}(0,1,1)$ proved to be the best.

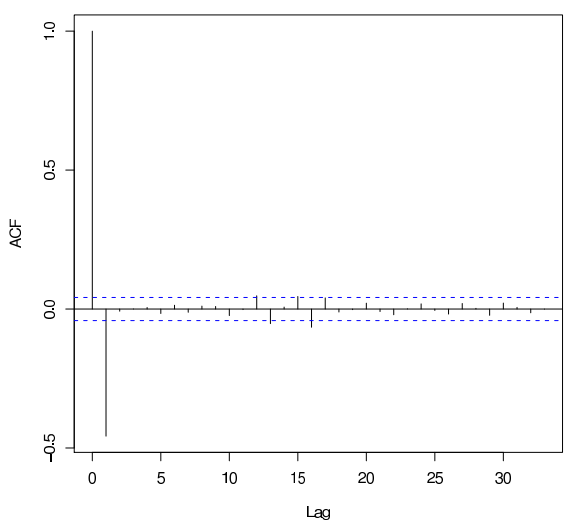

Fig. 2. The ACF of the differenced time series.

A typical example of the prediction model running to improve the raw estimates of load factor can be seen in figure 3 The figure plots the crude estimate and the improved estimate against the actual load factor (arising from the real traffic) which can only be observed at the router, not at the source. In this particular sample the load factor remains relatively constant and high at around 90 for the whole period examined. At almost all points in time the ARIMA modelling is closer to the real load factor than the crude estimate. More formal analysis of the modelling error will be given in the next section.

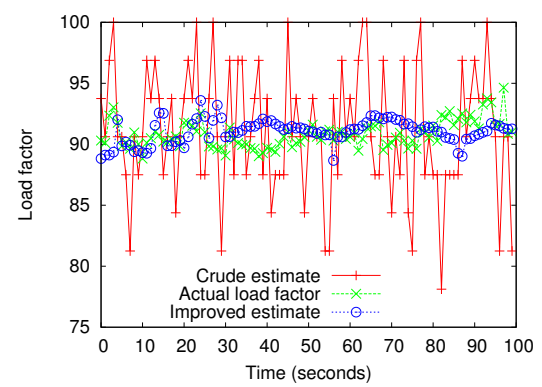

Fig. 3. An example of the ARIMA prediction.

\section{B. Estimation results}

The performance of $e_{i}$ and $\widehat{L_{i+1}}$ as estimators of $L_{i+1}$ can be compared against the bias of the estimate and the root mean square error (RMSE) of the prediction, which are for $N$ data points, $\sum_{i}\left(L_{i}-\widehat{L_{i}}\right) / N$ and $\sqrt{\sum_{i}\left(L_{i}-\widehat{L_{i}}\right)^{2} / N}$ respectively. The RMSE would be expected to change as a function of $t_{P}$ - as $t_{P}$ becomes smaller then $e_{i}$ is constructed from fewer ACKs and hence would be expected to be a more inaccurate estimate of $L_{i}$. However, the situation is not quite as clear as that since, if $t_{P}$ is small, $L_{i+1}$ might be expected to be very close in value to $L_{i}$ (since the load would not be expected to change too much over small time periods).

In this section, the simulation described in section [III is run for values of $t_{P}$ from 0.2 to 3.2 seconds. Each source produces estimates for the load $L_{i+1}$ using ARIMA $(0,1,1)$ and the RMSE (and bias) are measured for the crude and corrected estimators.

Figure 4 (top) shows for OC48 traffic the RMSE for the four queues outbound (left top) and inbound (right top). The dashed lines are the uncorrected results $e_{i}$ and the solid lines are the results corrected by the ARIMA process $\hat{L}_{i}$. As can be seen, the longer the sampling period, the better the estimate produced for the real load factor (for both raw and ARIMA estimates). In all cases, the ARIMA procedure produced an extremely noticeable reduction in the error. For all but the shortest sampling period the RMSE was below 10 in the corrected data which, given the range is $[0,100]$ the method is producing an extremely close prediction for the load factor in most sampled periods. Because of space constraints, graphs for bias are not produced here but in all routers for all time periods 

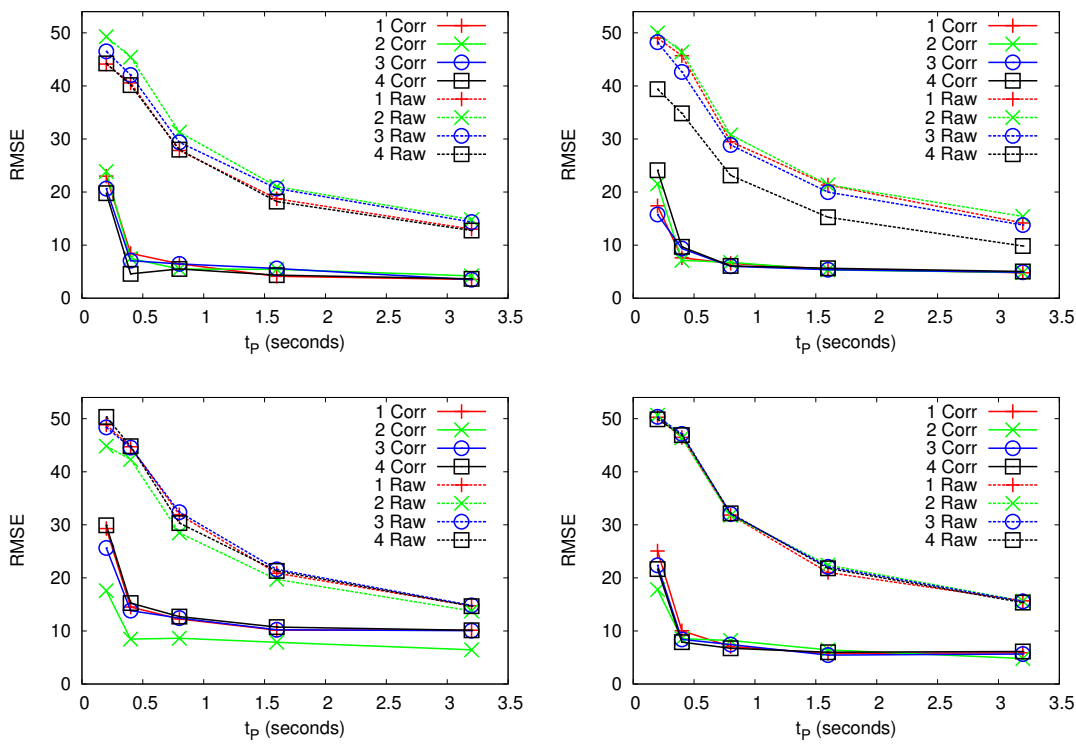

Fig. 4. Raw and corrected predictions RMSE for traffic originating from the left source (left) and the right source (right) for OC48 traffic (top) and OC12 traffic (bottom).

tested the bias was below five in magnitude and in the large majority of cases below one in magnitude. The conclusion is that bias is not a significant problem. As might be expected, the ARIMA modelling (which proceeds from the $e_{i}$ anyway) does not correct the bias.

Figure 4 (bottom) shows similar results for real traffic taken from the OC12 network and the topology in figure 1. The pattern is the same as for the OC48 traffic broadly speaking. The main differences is that the corrected RMSE is slightly larger for the data heading right. For the inbound data the RMSE (particularly uncorrected) is strikingly similar across all routers - the small differences in the data do not show up much in the plot. (This is thought to be the result of a single data stream being dominant in causing the congestion inbound and hence similar results on all routers.)

Tests were also run on the pre-signalling idea which, by getting a correct estimate of the number of routers on the path, greatly improved predictions. This is partly a result of the shortness of the path being tested here, five routers. Presignalling therefore led to an increase of more than six fold in the number of samples per time period to get each $e_{i}$. As would be expected the RMSE and absolute value of bias were decreased greatly as a result.

\section{CONCLUSION}

PCN is a new method for determining the load on any of a number (up to a fixed maximum) of intervening routers. Related methods exist in the literature but PCN has several advantages: 1) It produces an estimate of the load at each router. 2) It estimates the exact load rather than exactly determining whether the load is in one of a small number of load regions. 3) It requires the use of only one ECN bit.
The raw prediction results for PCN have relatively high errors in estimation of load factors but this can be corrected by using the time-series technique ARIMA modelling. This time series technique could also be useful for a variety of other probabilistic packet marking schemes.

The obvious future work would be to use the predicted load factors to produce an appropriate congestion response. It would also be of interest to implement the PCN protocol in a real kernel to see how it performs in a real life situation.

\section{REFERENCES}

1 V. Jacobson, "Congestion avoidance and control," in Proc. ACM/SIGCOMM, New York, NY, USA, 1988, pp. 314-329.

2 S. Floyd, "TCP and explicit congestion notification," SIGCOMM Comput. Commun. Rev., vol. 24, no. 5, pp. 8-23, 1994.

3 D. Katabi, M. Handley, and C. Rohrs, "Congestion control for high bandwidth-delay product networks," SIGCOMM Comput. Commun. Rev., vol. 32, no. 4, pp. 89-102, 2002

4 G. E. P. Box and G. M. Jenkins, Time Series Analysis, forecasting and control. Holden-Day, Inc., 1970.

5 K. K. Ramakrishnan, S. Floyd, and D. Black, "RFC3168: The addition of explicit congestion notification (ECN) to IP," September 2001

6 S. Athuraliya, H. Victor, L. Steven, H. Low, and Q. Yin, "REM: Active queue management," IEEE Network, vol. 15, no. 3, pp. 48-53, May-Jun 2001.

7 M. Adler, J. Cai, J. K. Shapiro, and D. F. Towsley, "Estimation of congestion price using probabilistic packet marking," in Proc. IEEE INFOCOM, Apr 2003.

8 R. W. Thommes and M. J. Coates, "Deterministic packet marking for congestion price estimation," in Proc. IEEE INFOCOM, Hong Kong, Mar 2004.

9 Y. Xia, L. Subramanian, I. Stoica, and S. Kalyanaraman, "One more bit is enough," IEEE/ACM Trans. Netw., vol. 16, no. 6, pp. 1281-1294, 2005.

10 J. Raj, K. Shiv, and V. Ram, "The OSU scheme for congestion avoidance in ATM networks: lessons learnt and extensions," Perform. Eval., vol. 31, no. 1-2, pp. 67-88, 1997.

11 F. Begtasevic and P. V. Mieghen, "Measurements of the hop count in the internet," in Passive and Active Measurement (PAM2001) Conf., Amsterdam, The Netherlands, Nov 2000. 